Pacific Journal of Mathematics

THE INVERSION THEOREM AND PLANCHERELS THEOREM

U. Huussano 


\section{THE INVERSION THEOREM AND PLANCHEREL'S THEOREM IN A BANACH SPACE}

\section{U. HAUSSMANN}

1. Introduction. Let $G$ be a locally compact abelian group with Haar measure $\mu$, and let $X$ be a complex Banach space and $C$ be the set of complex numbers. A classic theorem due to Plancherel ([8], [10]) states that the Fourier transform maps $L_{1}(G, C) \cap L_{2}(G, C)^{1}$ onto a dense subset of $L_{2}(\hat{G}, C)$ ( $\hat{G}$ is the dual group of $G$ and has Haar measure $m$ ) in such a way that $\int_{G} \alpha(g) \overline{\beta(g)} \mu(d g)=\int_{\hat{G}} \hat{\alpha}(\gamma) \overline{\widehat{\beta}(\gamma)} m(d \gamma)$ for all $\alpha, \beta$ in $L_{1}(G, C) \cap L_{2}(G, C)$ where $\hat{\alpha}$ is the Fourier transform of $\alpha$, given by $\hat{\alpha}(\gamma)=\int_{G} \overline{(g, \gamma)} \alpha(g) \mu(d g)$ for all $\gamma$ in $\hat{G}$. Here $(g, \gamma)$ denotes the action of the character $\gamma$ on $g$ in $G$. In this paper we extend this result to functions taking values in an inner product subspace of a Banach algebra.

Another well-known theorem ([8], [10]) states that if $\alpha$ is a positive definite element of $L_{1}(G, C) \cap L_{\infty}(G, C)$ then $\hat{\alpha}$ is in $L_{1}(\widehat{G}, C)$ and

$$
\alpha(g)=\int_{\hat{G}}(g, \gamma) \hat{\alpha}(\gamma) m(d \gamma)
$$

for (almost) all $g$ in $G$. This inversion theorem is also generalized to functions assuming values in certain admissible Banach spaces.

Our work relies heavily on an extension of Bochner's theorem established in [4]. We show that if $p$ is in $L_{1}(G, X) \cap L_{\infty}(G, X)$, if $p$ is positive definite (positivity is defined with respect to a particular cone in $X)$, and if $p(0)$ satisfies a certain finiteness condition, then $\hat{p}$, the Fourier transform of $p$, is in $L_{1}(\widehat{G}, X)$ and the inversion formula 1.1 given for $\alpha$ holds for $p$. A sharper theorem states that if $p$ is in $L_{1}(G, X) \cap L_{\infty}(G, X)$, if $p$ is positive definite, and if there is a real, finite, regular Borel measure $\lambda$ such that $\left\|\int_{G} \alpha(g) p(g) \mu(d g)\right\| \leqq$ $\int_{\hat{G}}|\hat{\alpha}(\gamma)| \lambda(d \gamma)$ for all $\alpha$ in $L_{1}(G, C)$, then $\hat{p}$ is in $L_{1}(\hat{G}, X)$ and 1.1 is satisfied by $p$.

Using this theory we extend to infinite dimensions some results due to Hewitt and Wigner ([7]).

1 For $1 \leqq p \leqq \infty L_{p}(G, X)$ is the space of $\mu$-measurable functions $f$ mapping $G$ into $X$. For $1 \leqq p<\infty$ we use the norm $\|\cdot\|_{p}$, where $\|f\|_{p}=\left\{\int_{G}\|f(g)\|^{p} \mu(d g)\right\}^{1 / p}$, and for $p=\infty$ we use the norm $\|f\|_{\infty}$ which is the $(\mu)$ essential supremum of $\|f(g)\|$ on $G$. $\|\cdot\|$ denotes the norm in $X$. 
2. Bochner's theorem and dominated functions. Let $X$ be a Banach space, $X^{*}$ the dual of $X$ and $X^{* *}$ the dual of $X^{*}$. For $\varphi$ in $X^{*}$ we denote the action of $\varphi$ on $x \in X$ by $(x, \varphi)$. Given a subset of $X^{*}$ we can define a cone of "positive" elements in $X$.

Definition 2.1. Let $\Phi$ be a subset of $X^{*}$. The subset $K_{\Phi}$ of $X$ given by

$$
K_{\Phi}=\{x \in X:(x, \varphi) \geqq 0 \text { for all } \varphi \in \Phi\}
$$

is called the cone determined by $\Phi$.

Sometimes we write simply $K$ if $\Phi$ is fixed by the context. $X_{\phi}$ is the set of "positive" elements.

Let $G$ be a $\sigma$-finite locally compact abelian group with Haar measure $\mu$ and let $\hat{G}$ be its dual group with Haar measure $m$.

Definition 2.3. Let $p$ be a map of $G$ into $X$. Then $p$ is $\Phi$ positive definite if it is measurable and if

$$
\sum_{n=1}^{N} \sum_{m=1}^{N} c_{n} \bar{c}_{m}\left(p\left(g_{n}-g_{m}\right), \varphi\right) \geqq 0
$$

for any integer $N$, any $c_{1}, \cdots, c_{N}$ in $C$, any $g_{1}, \cdots, g_{N}$ in $G$, and all $\phi$ in $\Phi$. If $p$ is in $L_{\infty}(G, X)$ the $p$ is integrally $\Phi$-positive definite if

$$
\left(\int_{G} \int_{G} \alpha(g) \overline{\kappa\left(g^{\prime}\right)} p\left(g-g^{\prime}\right) d \mu d \mu, \varphi\right) \geqq 0
$$

for all $\alpha$ in $L_{1}(G, C)$ and all $\varphi$ in $\Phi$.

Next we impose a condition which relates $\Phi$ to the topology of $X$.

Definition 2.6. The family $\Phi$ is full if there is a $\rho>0$ such that

$$
\|x\| \leqq \rho \sup \{\mid(x, \varphi)\|/\| \varphi \|: \varphi \in \Phi\}
$$

for all $x$ in $X$.

The following two propositions examine the relationship between the two notions of positive-definiteness.

Proposition 2.8. If $\Phi$ is full and $p$ is $\Phi$-positive definite then $p$ is in $L_{\infty}(G, X)$ and $p(0)$ is in $K_{\oplus}$.

Proof. It is readily shown that for $g$ in $G, \varphi$ in $\Phi,|(p(g), \varphi)| \leqq$ 
$(p(0), \varphi)$ so that $\|p(g)\| \leqq \rho\|p(0)\|$.

Proposition 2.9. Let $p$ be in $L_{\infty}(G, X)$ such that one version of $p$ is $\omega X$-continuous. ${ }^{2}$ Then $p$ is $\Phi$-positive definite iff $p$ is integrally $\Phi$-positive definite.

\section{Proof. See [4] or [6].}

We shall see shortly (Corollary 2.15) that all those elements of $L_{\infty}(G, X)$ of interest to us have the continuity required in Proposition 2.9 .

Next we recall some results from measure theory. Let $S$ be a locally compact topological space and let $\Sigma(S)$ be the Borel field of $S$ (i.e. the smallest $\sigma$-field containing the closed sets of $S$ ).

DEFINITION 2.10. A vector measure $\nu$ is a weakly countably additive set function defined on $\Sigma(S)$ and taking values in $X . \nu$ is weakly regular if the scalar measures $(\nu(\cdot), \varphi)$ are regular ${ }^{3}$ for all $\varphi$ in $X^{*}$. $\nu$ is $\Phi$-positive if $(\nu(E), \varphi) \geqq 0$ for all $\varphi$ in $\Phi$ and $E$ in $\Sigma(S)$.

Definition 2.11. A set function $\nu^{* *}$ mapping $\Sigma(S)$ into $X^{* *}$ is weak-*-regular if $\left(\varphi, \nu^{* *}(\cdot)\right)$ is a regular scalar measure for all $\varphi$ in $X^{*} . \quad \nu^{* *}$ is $\Phi$-positive if $\left(\varphi, \nu^{* *}(E)\right) \geqq 0$ for all $\varphi$ in $\Phi, E$ in $\Sigma(S)$.

If $\nu$ is a vector measure we denote its variation on a measurable set $E$ by $\|\nu\|(E)$ and its semi-variation by $|\nu|(E)$ ([2], [1]). The following theorem, an extension of Bochner's theorem, is essential to our work. The proof is given in [4]. We assume $\Phi$ is full.

THEOREM 2.12. (A) If $\nu$ is a weakly regular $\Phi$-positive vector measure defined on $\Sigma(\widehat{G})$ and if

$$
p(g)=\int_{\hat{G}}(g, \gamma) \nu(d \gamma)
$$

then $p$ is an integrally $\Phi$-positive definite element of $L_{\infty}(G, X)$.

(B) If $p$ is an integrally $\Phi$-positive definite element of $L_{\infty}(G, X)$, then there is a set function $\nu^{* *}$ mapping $\Sigma(\widehat{G})$ into $X^{* *}$ such that (i) $\nu^{* *}$ is weak-*-regular, $\Phi$-positive with finite semi-variation and (ii)

$$
(p(g), \varphi)=\int_{\hat{G}}(g, \gamma)\left(\varphi, \nu^{* *}(d \gamma)\right)
$$

for all $\varphi$ in $X^{*}$ and almost all $g$ in $G$.

2 The mapping $f$ of $G$ into $X$ is $\omega X$-continuous if it is continuous when the weak topology is imposed on $X$. $G$ retains its usual topology.

3 A scalar measure $\lambda$ is regular if, given $\varepsilon>0$ and $E \in \Sigma(S)$ with $\|\lambda\|(E)<\infty$ (i.e. $\lambda$ has finite variation on $E$ ), then there is a compact $K \subset E$ and an open $O \supset E$ such that $\|\lambda\|(O-K)<\varepsilon$. 
COROLlary 2.15. If $p$ is an integrally $\Phi$-positive definite element of $L_{\infty}(G, X)$ then one version of $p$ is $\omega X$-continuous. If $p$ is given by 2.13 , where $\nu$ is a weally regular $\Phi$-positive vector measure defined on $\Sigma(\hat{G})$, then $p$ is a continuous map of $G$ into $X$.

Proof. This follows from the relevant regularity. See also [6].

With the aid of Theorem 2.12 we could prove a useful inversion theorem. However, a different version of Bochner's theorem will allow us to establish a sharper theorem. We require first the following.

Definition 2.16. $p$ in $L_{\infty}(G, X)$ is dominated if there exists a finite, regular, positive Borel measure $\lambda$, such that

$$
\left\|\int_{G} \alpha(g) p(g) \mu(d g)\right\| \leqq \int_{\hat{G}}|\hat{\alpha}(\gamma)| \lambda(d \gamma)
$$

for all $\alpha$ in $L_{1}(G, C)$, where $\hat{\alpha}$ is the Fourier transform of $\alpha$, i.e. $\hat{\alpha}(\gamma)=\int_{G} \overline{(g, \gamma)} \alpha(g) \mu(d g)$. If $R^{+}$is the set of nonnegative real numbers, we have

Definition 2.18. Let $\Phi$ be a subset of $X$. Assume there is a function $\varphi_{0}$ mapping $K_{0}$ into $R^{+} \cup\{\infty\}$ in a linear manner such that $\varphi_{0}$ is uniformly positive on $K_{\Phi}$, i.e. there exists $k>0$ such that $k\left(x, \varphi_{0}\right) \geqq\|x\|$ for all $x$ in $K_{\phi}$. Furthermore assume there are at most countable sequences $\left\{c_{i}\right\}$ in $R^{+}$and $\left\{\varphi_{i}\right\}$ in $\Phi$ such that $\left(x, \varphi_{0}\right)=$ $\sum_{i=1}^{\infty} c_{i}\left(x, \varphi_{i}\right)$ for all $x$ in $K_{\varphi}$. Then we say that the pair $(\Phi, X)$ is admissible. We let $K_{0}=\left\{x \in K_{\varphi}:\left(x, \varphi_{0}\right)<\infty\right\}$.

LEMMA 2.19. If $(\Phi, X)$ is admissible, if $\Phi$ is full, and if $p \in$ $L_{\infty}(G, X)$ is integrally $\Phi$-positive definite with $p(0)$ in $K_{0}$, then $p$ is dominated.

In this lemma it is assumed we are talking about the $\omega X$ continuous version of $p(\cdot)$ (Corollary 2.15).

Proof. Let $\psi(\alpha)=\int_{G} \alpha(g) p(g) \mu(d g)$ for all $\alpha$ in $L_{1}(G, C)$, then $(\psi(\alpha), \varphi)=\int_{\hat{G}} \hat{\alpha}(\gamma)\left(\varphi, \nu^{* *}(d \gamma)\right)$ for some weak-*-regular, $\Phi$-positive set function $\nu^{* *}$ given by Theorem 2.12. We can actually define $\hat{\psi}(\cdot)$ mapping $C_{0}(\widehat{G})^{4}$ into $X$ by $(\hat{\psi}(f), \varphi)=\int_{\hat{G}} f(\gamma)\left(\varphi, \nu^{* *}(d \gamma)\right) .^{5}$ Then $\hat{\psi}$ is a

${ }_{4} C_{0}(\hat{G})$ is the space of continuous functions mapping $\hat{G}$ into $C$, which vanish at $\infty$ if $\hat{G}$ is only locally compact.

${ }_{5}$ For $\alpha$ in $L_{1}(G, C), \hat{\psi}(\hat{\alpha})=\psi(\alpha) \in X$. As $\left\{\hat{\alpha} \in C_{0}(\hat{G}): \alpha \in L_{1}(G, C)\right\}$ is dense in $C_{0}(\hat{G})$, and as $\hat{\psi}$ is continuous, it can be extended uniquely, with range in $X$. 
bounded linear map, $\|\hat{\psi}(f)\| \leqq\|f\|_{\infty}\left|\nu^{* *}\right|(\hat{G})$.

If $f$ is in $C_{0}(\hat{G})$ then $f=f_{1}-f_{2}+i f_{3}-i f_{4}$ where $f_{i}$ is in $C_{0}(\hat{G})$, $f_{i}(\gamma) \geqq 0$, and each pair of functions $\left(f_{1}, f_{2}\right),\left(f_{3}, f_{4}\right)$ has disjoint support. Hence $f_{i}(\gamma) \leqq|f(\gamma)|$, and $\hat{\psi}\left(f_{i}\right)$ is in $K_{\Phi}$ so that $\left\|\hat{\psi}\left(f_{i}\right)\right\| \leqq k\left(\hat{\psi}\left(f_{i}\right), \varphi_{0}\right)=$ $k \sum_{j=1}^{\infty} c_{j}\left(\hat{\psi}\left(f_{i}\right), \varphi_{j}\right)=k \sum_{j} c_{j} \int_{\hat{G}} f_{i}(\gamma)\left(\varphi_{j}, \nu^{* *}(d \gamma)\right)$. Consider now the set function $\lambda$ given by $\lambda(E)=\sum_{i=1}^{\infty} c_{i}\left(\varphi_{i}, \nu^{* *}(E)\right), E \in \Sigma(\widehat{G})$. Then $\lambda(E) \geqq 0$ for all $E$ in $\Sigma(\hat{G})$, and also $\lambda$ is additive. Moreover $\lambda(E) \leqq\left(p(0), \varphi_{0}\right)<$ $\infty$ as $p(0)$ is in $K_{0}$.

$\lambda$ is countably additive because $\lambda\left(\bigcup_{j} E_{j}\right)=\sum_{i} \sum_{j} c_{i}\left(\varphi_{i}, \nu^{* *}\left(E_{j}\right)\right)=$ $\sum_{j} \sum_{i} c_{i}\left(\varphi_{i}, \nu^{* *}\left(E_{j}\right)\right)=\sum_{j} \lambda\left(E_{j}\right)$, if the $E_{j}$ are disjoint (note that $c_{i}\left(\varphi_{i}\right.$, $\left.\nu^{* *}\left(E_{j}\right)\right) \geqq 0$ for all $i, j$ ). Also $\lambda$ is regular, for given $\varepsilon>0$ and $E$ in $\Sigma(\widehat{G})$, there is a number $N$ such that $\sum_{N+1}^{\infty} c_{i}\left(\varphi_{i}, \nu^{* *}(\widehat{G})\right)<\varepsilon / 2$ and there is a compact $K \subset E$ and an open $O \supset E$ such that $\left(\varphi_{i}, \nu^{* *}(0-\right.$ $K))<\varepsilon / 2 N c_{i}, i=1,2, \cdots, N$. Hence $\lambda(O-K)<\varepsilon$.

Then $\|\hat{\psi}(f)\| \leqq \sum_{i=1}^{4}\left\|\hat{\psi}\left(f_{i}\right)\right\| \leqq k \sum_{i} \int_{\hat{a}} f_{i}(\gamma) d \lambda \leqq 4 k \int_{\hat{G}}|f(\gamma)| d \lambda$. It follows that if $\lambda^{\prime}=4 k \lambda$ then $\|\psi(\alpha)\| \leqq \int_{\hat{G}}|\alpha(\gamma)| d \lambda^{\prime}$. This establishes the lemma.

We can now state the alternate version of Bochner's theorem. Assume $\Phi$ is full and countable

THEOREM 2.20. $p$ is a dominated, integrally $\Phi$-positive definite element of $L_{\infty}(G, X)$ iff there is a weakly regular $\Phi$-positive vector measure $\nu$ mapping $\Sigma(\widehat{G})$ into $X$ such that $\nu$ has finite variation, i.e. $\|\nu\|(\hat{G})<\infty$, and such that for any $\varphi$ in $X^{*}$,

$$
(p(g), \varphi)=\int_{\hat{G}}(g, \gamma)(\nu(d \gamma), \varphi),
$$

For the proof see [4]. Countability of $\Phi$ is not required for the only if part.

3. Inversion theorems. If $p \in L_{1}(G \cdot X)$ we recall that the Fourier transform of $p$ is given by

$$
\hat{p}(\gamma)=\int_{G} \overline{(g, \gamma)} p(g) \mu(d g) .
$$

For convenience we let $\mathscr{P}=\left\{p \in L_{\infty}(G, X): p\right.$ is integrally $\Phi$-positive definite $\}$ and $\mathscr{P}_{d}=(p \in \mathscr{P}: p$ is dominated $\}$. We recall that if $p \in \mathscr{P}$ then $p$ is $\omega X$-continuous (Corollary 2.15). If $(\Phi, X)$ is admissible then $\mathscr{T}_{0}$ is the set of functions $p$ mapping $G$ into $X$ such that $p$ is $\omega X$ continuous and such that $p(0)$ is in $K_{0}$ where $K_{0}$ is defined in 2.18.

Proposition 3.2. (A) If $p \in \operatorname{span}\left\{L_{1}(G, X) \cap \mathscr{P}\right\}$ and if $\varphi \in$ 
span $\{\Phi\}$ then $(\hat{p}(\cdot), \varphi) \in L_{1}(\hat{G}, C)$ and (B) if the Haar measure of $G$ is fixed then the Haar measure of $\hat{G}$ can be so normalized that

$$
(p(g), \varphi)=\int_{\hat{G}}(g, \gamma)(\hat{p}(\gamma), \varphi) m(d \gamma)
$$

is valid for all $p \in \operatorname{span}\left\{L_{1}(G, X) \cap \mathscr{P}\right\}$ and all $\varphi \in \operatorname{span}\{\Phi\}$.

Proof. It is evident the results need only hold for $p \in L_{1}(G, X) \cap$ $\mathscr{P}, \varphi \in \Phi$. But this follows from the scalar inversion theorem ([10], p. 22).

A better result is the following.

TheOREM 3.4. Assume $\Phi$ is full and $G$ is $\sigma$-finite. (A) If $p \in$ span $\left\{L_{1}(G, X) \cap \mathscr{P}_{d}\right\}$ then $\hat{p} \in L_{1}(\widehat{G}, X)$, and (B) with $\mu$ fixed, $m$ can be so normalized that for each $\varphi$ in $X^{*}$

$$
(p(g), \varphi)=\left(\int_{\hat{G}}(g, \gamma) \hat{p}(\gamma) m(d \gamma) \varphi\right) \quad \text { a.e.g. }
$$

If $\Phi$ is countable or if $p$ is continuous (3.5) becomes

$$
p(g)=\int_{\hat{G}}(g, \gamma) \hat{p}(\gamma) m(d \gamma)
$$

Proof. Again we need only prove the results for $p$ in $L_{1}(G, X) \cap$ $\mathscr{F}_{d}$. If $p$ is in $L_{1}(G, X)$ then $\hat{p}$ is in $C_{0}(\hat{G}, X)$, the space of continuous functions mapping $\hat{G}$ into $X$, which vanish at infinity if $\hat{G}$ is only locally compact but not compact. As $p$ is measurable and $G$ is $\sigma$ finite, $\hat{p}$ is essentially separably valued, and hence is measurable and a member of $L_{\infty}(\widehat{G}, X)$.

As $p$ is in $\mathscr{P}_{d}$, then by Theorem 2.20 there is a weakly regular $\Phi$-positive vector measure $\nu$ with finite variation such that for any $\varphi$ in $\Phi$

$$
\begin{aligned}
(p(g), \varphi) & =\int_{\hat{G}}(g, \gamma)(\nu(d \gamma), \varphi), & \quad a \cdot e \cdot g \\
& =\int_{\hat{G}}(g, \gamma)(\hat{p}(\gamma), \varphi) m(d \gamma) &
\end{aligned}
$$

by Proposition 3.2. As both integrals are continuous, the equality hold for all $g$. It follows, [10], that

$$
\begin{aligned}
(\nu(E), \varphi) & =\int_{E}(\hat{p}((\gamma), \varphi) m(d \gamma) \\
& =\left(\int_{E}(\hat{p}(\gamma) m(d \gamma), \varphi)\right.
\end{aligned}
$$


if $m(E)<\infty$, as $\hat{p}$ is bounded. Since $\Phi$ is full, we have

$$
\nu(E)=\int_{E} \hat{p}(\gamma) m(d \gamma)
$$

if $m(E)<\infty$. As $\hat{p}$ is in $C_{0}(\hat{G}, X)$ given $n$ these exists a compact set $K_{n}$ such that $\|\hat{p}(\gamma)\|<1 / n$ if $\gamma$ is in $\hat{G}-K_{n}$. Let $\chi_{n}(\cdot)$ be the indicator function of $K_{n}$. Then

$$
\begin{gathered}
\lim _{n \rightarrow \infty} \int_{\hat{G}}\left\|\chi_{n}(\gamma) \hat{p}(\gamma)\right\| m(d \gamma) \\
\quad=\lim _{n \rightarrow \infty} \int_{K_{n}}\|\hat{p}(\gamma)\| m(d \gamma) \\
\quad=\lim _{n \rightarrow \infty}\|\nu\|\left(K_{n}\right) \\
\quad=\|\nu\|(\hat{G})<\infty .
\end{gathered}
$$

Also $\left\|\chi_{n}(\gamma) \hat{p}(\gamma)\right\| \uparrow\|\hat{p}(\gamma)\|$ for each $\gamma$ in $\hat{G}$. Then by the monotone convergence theorem

$$
\lim _{n \rightarrow \infty} \int_{K_{n}}\|\hat{p}(\gamma)\| m(d \gamma)=\int_{\hat{G}}\|\hat{p}(\gamma)\| m(d \gamma) \leqq\|\nu\|(\hat{G}) .
$$

Hence $\hat{p}$ is in $L_{1}(\hat{G}, X)$, and for all measurable sets $E$,

$$
\nu(E)=\int_{E} \hat{p}(\gamma) m(d \gamma) .
$$

Since $\Phi$ is full (3.5) now follows from (3.7).

If $p$ is continuous, the set of measure zero where (3.5) does not hold is empty and (3.6) follows. If $\Phi$ is countable, the union of these null sets (one for each $\varphi$ in $\Phi$ ) is still a null set and again (3.6) holds.

Corollary 3.8. Assume $\Phi$ is full, $G$ is $\sigma$-finite, and $(\Phi, X)$ is admissible.

(A) If $p$ is in span $\left\{L_{1}(G, X) \cap \mathscr{P} \cap \mathscr{T}_{0}\right\}$ then $\hat{p}$ is in $L_{1}(\hat{G}, X)$.

(B) If $\mu$ is fixed, $m$ can be so normalized that for each $\emptyset$ in $X^{*}$ (3.5) holds. If $\Phi$ is countable or if $p$ is continuous then (3.6) holds.

Proof. Apply Lemma 2.19 and Theorem 3.4.

4. The Plancherel theorem. As usual this theorem is set in a Hilbert space, and so we must first develop the necessary structure. Assume now that $X$ is a Banach algebra with continuous involution $x \rightarrow x^{*}$.

Definition 4.1. The triplet $\left(\Phi, X, X_{0}\right)$ is strongly admissible if 
(i) $(\Phi, X)$ is admissible, (ii), $X_{0}$ is a non-trivial subspace of $X$ such that $x x^{*}$ is in $K_{0}{ }^{6}$ for all $x$ in $X_{0}$, and (iii) there exists $k_{0}>0$ such that if $x \in X_{0}$ then

$$
k_{0}\left\|x x^{*}\right\| \geqq\|x\|^{2},
$$

We note that 4.2 is satisfied if $X$ is a $C^{*}$-algebra. Now we have

Proposition 4.3. If $X$ is a Banach algebra and if $\left(\Phi, X, X_{0}\right)$ is strongly admissible then $X_{0}$ is a Hilbert space under the norm $\|\cdot\|_{0}$ where $\|x\|_{0}^{2}=\langle x, x\rangle_{0}$ and $\langle x, y\rangle_{0}=\left(x y^{*}, \varphi_{0}\right)$.

Proof. $\varphi_{0}$ is only defined on $K$ and we do not know that if $x, y \in X_{0}$ then $x y^{*} \in K$. However we can extend $\varphi_{0}$ by setting ( $x y^{*}$, $\left.\varphi_{0}\right)=\sum_{i=1}^{\infty} c_{i}\left(x y^{*}, \varphi_{i}\right)$ where $\left\{c_{i}\right\},\left\{\varphi_{i}\right\}$ define $\varphi_{0}$ on $K$. Then $\left|\langle x, y\rangle_{0}\right|=$ $\left|\left(x y^{*}, \varphi\right)\right|=\left|\sum_{i=1}^{\infty} c_{i}\left(x y^{*}, \varphi_{i}\right)\right| \leqq \sum_{1}^{\infty} c_{i}\left(x x^{*}, \varphi_{i}\right)^{1 / 2}\left(y y^{*}, \varphi_{i}\right)^{1 / 2}$ where the last inequality follows because $\varphi_{i}$ is a positive functional. Hence we can define $\langle x, y\rangle_{0}$ for $x, y \in X_{0}$ and $\left|\langle x, y\rangle_{0}\right| \leqq\|x\|_{0}\|y\|_{0}$. It follows from 2.18 and 4.2 that $k k_{0}\|x\|_{0}^{2} \geqq\|x\|^{2}$ and that $\|\cdot\|_{0}$ is a norm.

If $\left\{x_{n}\right\}$ is Cauchy in $\|\cdot\|_{0}$ then it is Cauchy in $\|\cdot\|$, so $x_{n} \rightarrow x \in X$. As $K$ is closed then $x x^{*} \in K$. Also $\left\{x_{n}\right\}$ is bounded in $\|\cdot\|_{0}$ because it is Cauchy, so $\sum_{i=1}^{\infty} c_{i}\left(x_{n} x_{n}^{*}, \varphi_{i}\right) \leqq M$, hence $\sum_{i=1}^{\infty} c_{i}\left(x x^{*}, \varphi_{i}\right) \leqq M$ or $x \in$ $K_{0}$. Choose $m(\varepsilon)$ such that if $n, m>m(\varepsilon)$ then $\left\|x_{n}-x_{m}\right\|_{0}<\varepsilon$. Then $\sum_{i=1}^{N} c_{i}\left(\left[x-x_{m}\right]\left[x-x_{m}\right]^{*}, \varphi_{i}\right)=\lim _{n \rightarrow \infty} \sum_{i=1}^{N} c_{i}\left(\left[x_{n}-x_{m}\right]\left[x_{n}-x_{m}\right]^{*}, \varphi_{i}\right) \leqq$ $\lim \sup _{n \rightarrow \infty} \sum_{i=1}^{\infty} c_{i}\left(\left[x_{n}-x_{m}\right]\left[x_{n}-x_{m}\right]^{*}, \varphi_{i}\right)<\varepsilon^{2}$ so that for $m>m(\varepsilon)$, $\left\|x-x_{m}\right\|_{0}<\varepsilon$, or $X_{0}$ is a Hilbert space.

If $X$ is a Banach algebra and $G$ is $\sigma$-finite, then $L_{1}(G, X)$ is also a Banach algebra ([5]). If $X$ has the involution $x \rightarrow x^{*}$, then we can define an involution on $L_{1}(G, X)$ as $p \rightarrow p^{*}$ where $p^{*}(g)=p(-g)$.* $^{*}$

THEOREM 4.4. If $G$ is $\sigma$-finite, $X$ is a Banach algebra with continuous involution, $\Phi$ is a full subset of $X^{*}$ and $\left(\Phi, X, X_{0}\right)$ is strongly admissible, then (i) if $\left\{e_{\alpha}\right\}$ is an orthonormal basis for $X_{0}$ and there exists $k_{1}$ such that $\left|\left\langle x, e_{\alpha}\right\rangle_{0}\right| \leqq k_{1}\|x\|$ for $x \in X_{0}$ and all $\alpha$, then the Fourier transform maps $L_{1}(G, X) \cap L_{2}\left(G, X_{0}\right)$ onto a dense subset of $L_{2}\left(\widehat{G}, X_{0}\right)$, (ii) for $q, r \in L_{1}(G, X) \cap L_{2}\left(G, X_{0}\right)$

$$
\int_{G} q(g) r(g) \mu(d g)=\int_{\hat{G}} \hat{q}(\gamma) \hat{r}(\gamma) m(d \gamma),
$$

(iii) for $q, r \in L_{1}(G, X) \cap L_{2}\left(G, X_{0}\right)$

$$
\langle q, r\rangle=\langle\hat{q}, \hat{r}\rangle,
$$

where $\langle q, r\rangle=\int_{G}\langle q(g), r(g)\rangle_{0} \mu(d g)$ and $\langle\hat{q}, \hat{r}\rangle=\int_{\hat{G}}\langle\hat{q}(\gamma), \hat{r}(\gamma)\rangle_{0} m(d \gamma)$.

$6 \quad K_{0}$ is defined in 2.18 . 
Proof. We shall put

$$
\|q\|_{1}=\int_{G}\|q(g)\| \mu(d g) \quad \text { and } \quad\|q\|_{2}=\left\{\int_{G}\|q(g)\|_{0}^{2} \mu(d g)\right\}^{1 / 2}
$$

for $q \in L_{1}(G, X) \cap L_{2}\left(G, X_{0}\right)$. Let $p(g)=\left(q * q^{*}\right)(g)$. As $q \in L_{1}(G, X)$ so is $p$ with $\|p\|_{1} \leqq\|q\|_{1}^{2}$. It can also be shown that $p \in C_{0}\left(G, X_{0}\right)$ as $q \in L_{2}\left(G, X_{0}\right)$. Now $p(0)=\int_{G} q(g) q(g)^{*} \mu(d g) \in K$ so

$$
\begin{aligned}
\left(p(0), \varphi_{0}\right) & =\left(\int_{G} q(g) q(g)^{*} \mu(d g), \varphi_{0}\right) \\
& =\sum_{i=1}^{\infty} c_{i} \int_{G}\left(q(g) q(g)^{*}, \varphi_{i}\right) \mu(d g) \\
& =\int_{G}\left(q(g) q(g)^{*}, \varphi_{0}\right) \mu(d g) \\
& =\int_{G}\|q(g)\|_{0}^{2} \mu(d g) \\
& =\|q\|_{2}^{2}<\infty
\end{aligned}
$$

using the monotone convergence theorem. Hence $p \in L_{1}(G, X) \cap \mathscr{T}_{0}$.

Now $C_{0}\left(G, X_{0}\right) \subset C_{0}(G, X)$ so $p \in L_{\infty}(G, X)$. Also

$$
\begin{aligned}
\int_{G} \int_{G} & \alpha(g) \overline{\alpha\left(g^{\prime}\right)} p\left(g-g^{\prime}\right) \mu(d g) \mu\left(d g^{\prime}\right) \\
& =\int_{G}\left[\int_{G} \alpha(g) q\left(g-g^{\prime \prime}\right) \mu(d g)\right]\left[\int_{G} \alpha\left(g^{\prime}\right) q\left(g^{\prime}-g^{\prime \prime}\right) \mu\left(d g^{\prime}\right)\right]^{*} \mu\left(d g^{\prime \prime}\right) \\
& =\int_{G} q^{\prime}(g) q^{\prime}(g)^{*} \mu(d g)
\end{aligned}
$$

using the Fubini and Tonelli theorems with $\alpha \in L_{1}(G, C)$, where $q^{\prime}=$ $\alpha * q \in L_{2}\left(G, X_{0}\right) \quad$ ([5]) so $q^{\prime}(g) \in X_{0}$ a.e. or $q^{\prime}(g) q^{\prime}(g)^{*} \in K_{0}$ a.e. Hence if $\phi \in \Phi$ then

$$
\left(\int_{G} q^{\prime}(g) q^{\prime}(g)^{*} \mu(d g), \varphi\right)=\int_{G}\left(q^{\prime}(g) q^{\prime}(g)^{*}, \varphi\right) \mu(d g) \geqq 0
$$

or $p \in \mathscr{P}$.

Consequently Corollary 3.8 yields $p(g)=\int_{\hat{G}}(g, \gamma) \hat{p}(\gamma) m(d \gamma)$. Then

$$
\begin{aligned}
\infty>\|q\|_{2}^{2} & =\langle q, q\rangle=\sum_{i=1}^{\infty} c_{i}\left(p(0), \varphi_{i}\right) \\
& =\sum_{i} c_{i} \int_{\hat{G}}\left(\hat{p}(\gamma), \varphi_{i}\right) m(d \gamma) \\
& =\int_{\hat{G}}\left(\hat{p}(\gamma), \varphi_{0}\right) m(d \gamma)=\langle\hat{q}, \hat{q}\rangle .
\end{aligned}
$$

We have used the monotone convergence theorem again. Hence the 
Fourier transform maps into $L_{2}\left(\hat{G}, X_{0}\right)$. By the usual expansion $\langle q, r\rangle=\langle\hat{q}, \hat{r}\rangle$. This establishes (iii).

Moreover $\int_{G} q(g) q(g)^{*} \mu(d g)=p(0)=\int_{\hat{G}} \hat{p}(\gamma) m(d \gamma)=\int_{\hat{G}} \hat{q}(\gamma) \hat{q}(\gamma)^{*} m(d \gamma)$. Also if $x, y$ are elements of a Banach algebra with involution then

$$
\begin{aligned}
4 x y^{*}= & (x+y)(x+y)^{*}-(x-y)(x-y)^{*} \\
& +i(x+i y)(x+i y)^{*}-i(x-i y)(x-i y)^{*}
\end{aligned}
$$

so that (ii) is also proved.

We need only show that $Q=\left\{\hat{q} \in L_{2}\left(\hat{G}, X_{0}\right): q\right.$ in $\left.L_{1}(G, X) \cap L_{2}\left(G, X_{0}\right)\right\}$ is dense in $L_{2}\left(\hat{G}, X_{0}\right)$. As $\mu$ is translation invariant so is $L_{1}(G, X) \cap$ $L_{2}\left(G, X_{0}\right)$ and hence $Q$ is invariant under multiplication by $(g, \cdot)$ for any $g \in G$. If $r \in L_{2}\left(\widehat{G}, K_{0}\right)$ and $\langle q, r\rangle=0$ for all $q \in Q$, then $\int_{\hat{\sigma}}\left(q(\gamma) r(\gamma)^{*}, \varphi_{0}\right)(g, \gamma) m(d \gamma)=0$ for all $q \in Q$ and $g \in G$. As $\left(q(\cdot) r(\cdot)^{*}\right.$, $\left.\mathscr{\varphi}_{0}\right) \in L_{1}(\hat{G}, C)$ it follows that $\left(q(\gamma) r(\gamma)^{*}, \varphi_{0}\right)=0$ a.e. for every $q \in Q$, or $\langle q(\gamma), r(\gamma)\rangle_{0}=0$ a.e. As $L_{1}(G, X) \cap L_{2}\left(G, X_{0}\right)$ is invariant under multiplication by $(\cdot, \gamma), \gamma \in \hat{G}$, then $Q$ is invariant under translation. ${ }^{7}$ Hence to every $\gamma_{0} \in \hat{G}$ there corresponds $q_{0} \in Q$ such that $q_{0}\left(\gamma_{0}\right) \neq 0$, so $q_{0}(\gamma) \neq 0$ in a neighborhood of $\gamma_{0}$ as $q_{0}$ is continuous. If $\left\{e_{\alpha}\right\}$ is the basis of $X_{0}$ mentioned in the statement of part (i), then $q_{0}(\cdot)=\sum_{\alpha} q_{\alpha}(\cdot) e_{\alpha}$ so there exists $\alpha_{0}$ such that $q_{\alpha_{0}}(\gamma) \neq 0$ in a neighborhood of $\gamma_{0}$. If $q_{0}(\cdot)=\hat{p}(\cdot)$ then $p=\sum_{\alpha} p_{\alpha} e_{\alpha}$ and as $p \in L_{2}\left(G, X_{0}\right), p_{\alpha} \in L_{2}(G, C)$. By hypothesis $\left|\left\langle x, e_{\alpha}\right\rangle_{0}\right| \leqq k_{1}\|x\|$ so $p_{\alpha} \in L_{1}(G, C)$ and $\hat{p}_{\alpha}(\gamma)=q_{\alpha}(\gamma)$. Hence $p_{\alpha_{0}}(\cdot) e_{\alpha} \in$ $L_{1}(G, X) \cap L_{2}\left(G, X_{0}\right)$ for any $\alpha$ and $\hat{p}_{\alpha_{0}}(\cdot) e_{\alpha}=q_{\alpha_{0}}(\cdot) e_{\alpha} \in Q$. Since for each $\gamma$ in a neighborhood of $\gamma_{0},\left\{q_{\alpha_{0}}(\gamma) e_{\alpha}\right\}_{\alpha}$ forms a complete set in $X_{0}$, and since $0=\left\langle q_{\alpha_{0}}(\gamma) e_{\alpha}, r(\gamma)\right\rangle_{0}$, then $r(\gamma)=0$ in a neighborhood of $\gamma_{0}$. But $\gamma_{0}$ was arbitrary so $r=0$, or $Q$ is orthogonal only to 0 in $L_{2}\left(\widehat{G}, X_{0}\right)$, a Hilbert space. Hence $Q$ is dense in $L_{2}\left(\widehat{G}, X_{0}\right)$. This completes the proof.

CoRollary 4.8. Under the assumptions of the theorem the Fourier transform can be extended in a unique manner to an isometry of $L_{2}\left(G, X_{0}\right)$ onto $L_{2}\left(\hat{G}, X_{0}\right)$.

Proof. We need only show $L_{1}(G, X) \cap L_{2}\left(G, X_{0}\right)$ is dense in $L_{2}(G$, $\left.X_{0}\right)$. But $C_{c}\left(G, X_{0}\right)^{8}$ is dense in $L_{2}\left(G, X_{0}\right)([6])$. Hence if $f \in L_{2}\left(G, X_{0}\right)$ then there exists $\left\{f_{n}\right\}_{1}^{\infty} \subset C_{c}\left(G, X_{0}\right) \cap L_{2}\left(G, X_{0}\right)$ such that $\left\|f_{n}-f\right\|_{2} \rightarrow 0$. Then $f_{n} \in C_{c}(G, X)$ and $f_{n}$ is measurable so $f_{n} \in L_{1}(G, X)$.

REMARK. The equality (4.5) holds for all $q, r \in L_{2}\left(G, X_{0}\right)$. Moreover, all results are correct assuming only that $\varphi_{0}$ is an arbitrary

7 By this we mean that $f_{\gamma_{0}}$ is in $Q$ for any $\gamma_{0}$ in $\hat{G}$ if $f$ is in $Q$ and $f_{\gamma_{0}}(\gamma)=f\left(\gamma+\gamma_{0}\right)$.

$8 C_{c}\left(G, X_{0}\right)$ denotes the set of functions in $C_{0}\left(G, X_{0}\right)$ having compact support. 
linear combination of $\varphi_{i}^{\prime}$ 's, i.e. $\varphi_{0}=\sum_{\alpha \in A} c_{\alpha} \varphi_{\alpha}$.

5. Examples. Here we give some examples of admissible pairs and strongly admissible triplets.

ExAmple 5.1. Let $X=L_{1}([0,1], C)$ so $X$ is weakly complete, and let $\Phi$ consist of elements $\varphi_{i}$ such that

$$
\left(x, \varphi_{i}\right)=\int_{0}^{1} \chi_{i}(t) x(t) d t \quad x \in X
$$

where $\chi_{i}(\cdot)$ is the indicator function of one of a countable collection of sets $\left\{E_{i}\right\}$ dense in $\Sigma([0,1])$ under the usual Hausdorff metric. Assume $E_{1}=[0,1]$. Then it can be shown ([4], [6]) that $\Phi$ is full and that $K$ is the cone of nonnegative (a.e.) functions. Let $\left(x, \varphi_{0}\right)=$ $\left(x, \varphi_{1}\right)=\int_{0}^{1} x(s) d s=\|x\|_{1}$ for $x \in K$. Hence $(\Phi, X)$ is admissible and $K_{0}=K$.

If $p$ is in $\mathscr{P}$ then $p(0)$ is in $K=K_{0}$ by Propositions 2.8 and 2.9 and by Corollary 2.15. So $p \in \mathscr{T}_{0}$ and the inversion theorem states that if $p \in \operatorname{sp}\left\{L_{1}\left(G, L_{1}([0,1], C)\right) \cap \mathscr{P}\right\}$ then $\hat{p} \in L_{1}\left(\hat{G}, L_{1}([0,1], C)\right)$ and $p(g)=\int_{\hat{G}}(g, \gamma) \hat{p}(\gamma) m(d \gamma)$.

The author does not know of any nontrivial subspace $X_{0}$ which would make $\left(\Phi, X, X_{0}\right)$ strongly admissible.

ExAmple 5.3. Let $X=H$, a separable Hilbert space with a fixed orthonormal basis $\left\{e_{i}\right\}_{1}^{\infty}$. Let $H_{0}$ be the set of elements of $H$ with all but a finite number of components zero, with nonzero components being real, rational nonnegative, and with norm less than or equal to one. Then $\Phi=H_{0}$ is full ([4], [6]) and countable and $K_{\Phi}=\{h \in$ $\left.H: h_{i} \geqq 0\right\} .^{9} \quad$ Let $\left(h, \varphi_{i}\right)=\left\langle h, e_{i}\right\rangle, i=1,2, \cdots$ and $\varphi_{0}=\sum_{1}^{\infty} \varphi_{i}$. Then $\varphi_{0}$ maps $K$ into $[0, \infty]$, and for $h$ in $K$

$$
\left(h, \varphi_{0}\right)^{2}=\left(\Sigma h_{i}\right)^{2} \geqq \Sigma h_{i}^{2}=\|h\|^{2}
$$

so that $(\Phi, H)$ is admissible and $K_{0}=\left\{h \in K: \sum_{1}^{\infty} h_{i}<\infty\right\}$.

$H$ becomes a Banach algebra if we define $h k=\sum_{i}^{\infty} h_{i} k_{i} e_{i}$. Let $h^{*}=\Sigma \bar{h}_{i} e_{i}$. For $h$ in $H h h^{*}$ is in $K$ and $\left(h h^{*}, \varphi_{0}\right)=\sum_{i} h_{i} \bar{h}_{i}=\|h\|^{2}$. We do not have $k\left\|h h^{*}\right\| \geqq\|h\|^{2}$ for some $k>0$, but we do have $\|h\|_{0}=\|h\|$ which is sufficient to show that $X_{0}=H$. Hence $(\Phi, H, H)$ is "strongly admissible," and the Plancherel theorem applies. Note that the condition $\left|\left\langle h, e_{i}\right\rangle\right| \leqq\|h\|$ also holds.

ExAmple 5.4. Let $X=\mathscr{L}(H, H)$, the linear bounded operators 
mapping the separable Hilbert space $H$ into itself. Let $H_{0}$ be a countable dense subset of the unit ball in $H$ and let $\Phi=\left\{\phi \in X^{*}\right.$ : $\left.(T, \varphi)=\langle T h, h\rangle, T \in \mathscr{L}(H, H), h \in H_{0}\right\}$. Let $\left\{e_{i}\right\}$ also be in $H_{0}$ for some orthonormal basis $\left\{e_{i}\right\}$. Then $\Phi$ is full and countable and $K_{\Phi}$ is the cone of positive operators ([4] or [6]). Let $\left(T, \varphi_{0}\right)=\sum_{1}^{\infty}\left\langle T e_{i}, e_{i}\right\rangle$. So $\varphi_{0}=\sum_{1}^{\infty} \varphi_{i}$ is the trace, where $\left(T, \varphi_{i}\right)=\left\langle T e_{i}, e_{i}\right\rangle$. Then $\varphi_{0}: K \rightarrow$ $[0, \infty],\left(T, \varphi_{0}\right)=\operatorname{tr} T \geqq\|T\|$ if $T$ is positive. Hence $(\Phi, \mathscr{L}(H, H))$ is admissible and $K_{0}$ is the cone of positive operators of finite trace and so a subset of the trace class.

We can see that in one case the condition $p \in \mathscr{T}_{0}$ is necessary for the inversion theorem to hold. Let $G$ be the circle group so that $\hat{G}$ is countable. Label its elements $\gamma_{1}, \gamma_{2}, \cdots$, and let the set function $\nu$ be given by

$$
\left\langle\nu\left(\left\{\gamma_{n}\right\}\right) e_{i}, e_{j}\right\rangle=p_{n} \delta_{n i} \delta_{n j},{ }^{10} \quad i, j, n=1,2, \cdots
$$

where $\infty>M \geqq p_{n} \geqq 0$. $\quad \nu$ can be extended to a countably additive measure of finite semi-variation in the obvious way. Let $p$ be given by

$$
p(t)=\sum_{n=1}^{\infty} e^{i t \gamma_{n}} \boldsymbol{\nu}\left(\left\{\gamma_{n}\right\}\right) .
$$

Then $p$ is in $\mathscr{P}$ (Theorem $2.12(\mathrm{~A})$ ) and $p$ is in $L_{1}(G, X)$ because $G$ is compact and $\|p(t)\| \leqq M$. If $\hat{p}$ is to be in $L_{1}(\hat{G}, X)$ then $\|\nu\|(\widehat{G})$ must be finite or $\sum_{1}^{\infty} p_{n}=\operatorname{tr} p(0)<\infty$.

Finally let $X_{0}=\mathscr{T}$, the Hilbert-Schmidt operators ([3]). Then for $T$ in $\mathscr{N}, T T^{*}$ is in the trace class and is positive so that $T T^{*}$ is in $K_{0}$. Also $\mathscr{L}(H, H)$ is a $C^{*}$-algebra so $(\Phi, \mathscr{L}(H, H), \mathscr{N})$ is strongly admissible. A basis for $\mathscr{N}$ is given by $\left\{T_{i j}\right\}$ where $\left\langle T_{i j} e_{k}\right.$, $\left.e_{l}\right\rangle=\delta_{i k} \delta_{j l}, k, l=1,2, \cdots$. Then $\left|\left\langle T, T_{i j}\right\rangle_{0}\right|=\left|\left\langle T e_{i}, e_{j}\right\rangle\right| \leqq\|T\|$, and the condition in (i) of Theorem 4.4 also holds.

6. Fourier transforms on representations. In this section we apply the preceding theory to extend the inversion theorem and Plancherel's theorem to "Fourier transforms" defined for unitary representations in a separable Hilbert space. The case where $H$ is finite dimensional has been treated by Hewitt and Wigner [7]. Let $H$ be a separable complex Hilbert space, and let $U(\cdot)$ be a continuous unitary representation of $G$ in $\mathscr{L}(H, H)$, i.e. $U\left(g+g^{\prime}\right)=U(g) U\left(g^{\prime}\right)$, $U(0)=I$, and $V$ is a continuous mapping of $G$ into the unitary operators on $H$. It follows [9] that there exists a sequence $\left\{\gamma_{i}\right\}$ of characters, and a resolution $\left\{\pi_{i}\right\}$ of the identity in $\mathscr{L}(H, H)$, such that

$10 \quad \delta_{n i}$ is the Kronecker delta. 


$$
U(g)=\sum_{i}\left(g, \gamma_{i}\right) \pi_{i}
$$

(The summation is at most countable). If $p$ is in $L_{1}(G, \mathscr{L}(H, H)$ ) define the transform

$$
\hat{p}(U)=\int_{G} p(g) U(-g) \mu(d g) .
$$

We shall first consider the question of invertibility of this transform. As we shall see, it suffices to know $\hat{p}(U)$ for all $U$ corresponding to a fixed resolution $\left\{\pi_{i}\right\}$.

From now on consider $\left\{\pi_{i}\right\}$ fixed, and let us denote the set of subscripts by $S$. Then $S$ is at most countable, $\sum_{i \in S} \pi_{i}=I$. Define $\mathscr{R} \equiv \prod_{i \in S} \widehat{G}_{i}$, where $\widehat{G}_{i} \equiv \widehat{G}$ for all $i$, with the product topology. Then $\mathscr{R}$ can be considered as the set of all representations corresponding to $\left\{\pi_{i}\right\}$, if we put

$$
r \longleftrightarrow \sum_{i \in S}\left(\cdot, \gamma_{i}\right) \pi_{i}=U(\cdot)
$$

whenever $r=\left\{\gamma_{i}\right\} \in \mathscr{R}$.

Let us now introduce a measure on $\mathscr{R}$. Choose a symmetric neighborhood $A$ of 0 in $\hat{G}$ such that the closure of $A$ is compact. Hence $0<m(A)<\infty$. Assume $m$ is normalized (relative to $\mu$ ) such that the inversion theorem holds. Now normalize $\mu$ such that $m(A)=1$. Note that if $G$ is discrete and $A=\hat{G}$, or if $G$ is compact and $A=\{0\}$, then the usual normalizations of $\mu$ and $m$ occur. For $\alpha$ in $\widehat{G}$ and $E$ in $\Sigma(\widehat{G})$ define

$$
m_{\alpha}(E)=m[E \cap(A+\alpha)] .
$$

Then $m_{\alpha}(\cdot)$ is a probability measure on $\hat{G}$, and by the Kolmogorov extension theorem, there exists a unique probability measure

$$
m_{\alpha}^{\infty}=m_{\alpha} \times m_{\alpha} \times \cdots
$$

on $\mathscr{R}$. We set $\mathscr{R}^{i}=\prod_{j \in S-\{i\}} \hat{G}_{j}$. For $E$ in $\Sigma\left(\mathscr{R}^{i}\right)$ write

$$
m_{\alpha}^{i}(E)=\int_{\Omega} \chi_{E \times \hat{G}}(r) m_{\alpha}^{\infty}(d r)
$$

where it is understood we are integrating out $\gamma_{i}$.

Now assume $G$ is $\sigma$-finite and $\Phi$ is a full, countable subset of $\mathscr{L}(H, H)^{*}$. With the previous notation we have

THEOREM 6.4. If $p$ is in span $\left\{L_{1}(G, \mathscr{L}(H, H)) \cap \mathscr{P}_{d}\right\}$, then

$$
p(g)=\int_{\hat{G}} \int_{\mathscr{R}} \hat{p}(U) U(g) m_{\alpha}^{\infty}(d r) m(d \alpha) .
$$


Proof. $\pi_{i}$ is a projection on the subspace $H_{i}$ of $H$. Moreover if we consider the equivalent spectral representation ([9], p. 247), then the subspaces are mutually orthogonal. Let us write $f(\alpha)=\hat{p}(\alpha)(g, \alpha)$, and $f^{j}(r) \equiv f\left(\gamma_{j}\right)$ when $r=\left\{\gamma_{i}\right\}$. Then for $n$ finite, $\beta \in \widehat{G}$ and $h$ in $H$,

$$
\begin{aligned}
& \left\|\sum_{i=1}^{n} \int_{i=2} f^{i}(r) m_{\beta}^{\infty}(d r) \pi_{i} h\right\| \\
& =\left\|\sum_{i=1}^{n} \int_{\hat{G}} \int_{\boldsymbol{\Omega}_{i} i} f(\alpha) m_{\beta}^{i}(d r) m_{\beta}(d \alpha) \pi_{i} h\right\| \\
& \quad=\left\|\sum_{i=1}^{n} \int_{\hat{\hat{G}}} f(\alpha) m_{\beta}(d \alpha) \pi_{i} h\right\| \\
& \quad \leqq\left\|\int_{\hat{G}} f(\alpha) m_{\beta}(d \alpha)\right\|\|h\|
\end{aligned}
$$

so that

$$
\begin{aligned}
\left\|\sum_{i=1}^{n} \int_{\mathscr{R}} f_{i}(r) m_{\beta}^{\infty}(d r) \pi_{i}\right\| & \leqq\left\|\int_{\hat{G}} f(\alpha) m_{\beta}(d \alpha)\right\| \\
& \leqq \int_{\hat{G}}\|\hat{p}(\alpha)\| \chi_{A+\beta}(\alpha) m(d \alpha) .
\end{aligned}
$$

As $\hat{p}$ is in $L_{1}$, and as $m(A)=1$, then

$$
\begin{gathered}
\int_{\hat{G}} \int_{\hat{G}}\|\hat{p}(\alpha)\| \chi_{A+\beta}(\alpha) m(d \alpha) m(d \beta) \\
=\|\hat{p}\|_{1} .
\end{gathered}
$$

Hence

$$
\begin{aligned}
\sum_{i \in S} \int_{\hat{G}} \int_{\mathscr{R}} f^{i}(r) m_{\beta}^{\infty}(d r) m(d \beta) \pi_{i} \\
\quad=\int_{\hat{G}} \sum_{i \in S} \int_{\mathscr{R}} f^{i}(r) m_{\beta}^{\infty}(d r) \pi_{i} m(d \beta) .
\end{aligned}
$$

Moreover

$$
\begin{aligned}
\int_{\mathscr{R}} f^{i}(r) m_{\beta}^{\infty}(d r) \pi_{i} \\
\quad=\int_{\mathscr{R}} \hat{p}\left(\gamma_{i}\right)\left(g, \gamma_{i}\right) m_{\beta}^{\infty}(d r) \pi_{i} \\
=\int_{\mathscr{R}} \int_{G} p\left(g^{\prime}\right)\left(g-g^{\prime}, \gamma_{i}\right) \mu\left(d g^{\prime}\right) m_{\beta}^{\infty}(d r) \pi_{i},
\end{aligned}
$$

and

$$
\begin{aligned}
& \left\|\sum_{i=1}^{n} p\left(g^{\prime}\right)\left(g-g^{\prime}, \gamma_{i}\right) \pi_{i} h\right\| \\
& \quad \leqq\left\|p\left(g^{\prime}\right)\right\|\left\|\sum_{i=1}^{n}\left(g-g^{\prime}, \gamma_{i}\right) \pi_{i} h\right\| \\
& \quad \leqq\left\|p\left(g^{\prime}\right)\right\|\|h\|
\end{aligned}
$$


as $|(g, \gamma)|=1$ and the $\pi_{i}$ 's are orthogonal projections. As $p$ is in $L_{1}$, and as $m_{\beta}^{\infty}(\mathscr{R})=1$, then

$$
\begin{aligned}
\sum_{i \in S} \int_{\mathscr{R}} f_{i}(r) m_{\beta}^{\infty}(d r) \pi_{i} \\
\quad=\int_{\mathscr{R}} \int_{G} \sum_{i \in S} p\left(g^{\prime}\right)\left(g-g^{\prime}, \gamma_{i}\right) \pi_{i} \mu\left(d g^{\prime}\right) m_{\beta}^{\infty}(d r) .
\end{aligned}
$$

On the other hand

$$
\begin{aligned}
\hat{p}(U) U(g) & =\int_{G} p\left(g^{\prime}\right) U\left(-g^{\prime}\right) \mu\left(d g^{\prime}\right) U(g) \\
& =\int_{G} p\left(g^{\prime}\right) U\left(g-g^{\prime}\right) \mu\left(d g^{\prime}\right) \\
& =\int_{G} p\left(g^{\prime}\right) \sum_{i \in S}\left(g-g^{\prime}, \gamma_{i}\right) \pi_{i} \mu\left(d g^{\prime}\right) .
\end{aligned}
$$

Hence we have shown that for each $\beta, g, \hat{p}(U) U(g)$ is integrable $m_{\beta}^{\infty}(d r)$, and $\int_{\mathscr{R}} \hat{p}(U) U(g) m_{\beta}^{\infty}(d r)$ is integrable $m(d \beta)$, so that 6.5 makes sense.

Finally

$$
\begin{aligned}
\int_{\hat{G}} \int_{\mathscr{R}} & \hat{p}(U) U(g) m_{\beta}^{\infty}(d r) m(d \beta) \\
& =\sum_{i \in S} \int_{\hat{G}} \int_{\mathscr{R}} f^{i}(r) m_{\beta}^{\infty}(d r) m(d \beta) \pi_{i} \\
& =\sum_{i \in S} \int_{\hat{G}} \int_{\hat{G}} \int_{\overparen{\Omega} i} \hat{p}(\alpha)(g, \alpha) m_{\beta}^{i}(d r) m_{\beta}(d \alpha) m(d \beta) \pi_{i} \\
& =\sum_{i \in S} \int_{\hat{G}} \int_{\hat{G}} \hat{p}(\alpha)(g, \alpha) m_{\beta}(d \alpha) m(d \beta) \pi_{i} \\
& =\sum_{i \in S} \int_{\hat{G}} \int_{\hat{G}} \chi_{A}(\alpha-\beta) \hat{p}(\alpha)(g, \alpha) m(d \beta) m(d \alpha) \pi_{i} \\
& =\sum_{i \in S} \int_{\hat{G}} \hat{p}(\alpha)(g, \alpha) m(d \alpha) \pi_{i} \\
& =\sum_{i \in S} p(g) \pi_{i} \\
& =p(g) .
\end{aligned}
$$

We have made use of $6.6,6.7,6.8$, and the inversion theorem. The theorem is established.

Now consider the setting of Example 5.4.

THEOREM 6.9. If $p$ and $q$ are in $L_{1}\left[G_{1}, \mathscr{L}[H, H)\right] \cap L_{2}(G, \mathscr{N})$, then

$$
\int_{G} p(g) q(g)^{*} \mu(d g)=\int_{\hat{G}} \int_{\mathscr{R}} \hat{p}(U) \hat{q}(U)^{*} m_{\alpha}^{\infty}(d r) m(d \alpha)
$$


Proof. The proof is similar to the previous one except that Theorem 4.4 is used.

\section{Further applications of this theory can be found in [6].}

\section{REFERENCES}

1. N. Dinculeanu, Vector Measures, Deutscher Verlag der Wissenchafft, Berlin, 1966.

2. N. Dunford, J. Schwartz, Linear Operators, Part I, Interscience, New York, 1958.

3. - Linear Operators, Part II, Interscience, New York, 1963.

4. P. L. Falb, and U. G. Haussmann, Bochner's theorem in in finite dimensions, Pacific J. Math., 43 (1972), 601-618.

5. P. L. Falb, M. I. Freedman, A generalized transform theory for causal operators, SIAM J. on Control, 7 (1969), 452-471.

6. U. G. Haussmann, Harmonic Analysis in Banach Space, Ph. D. thesis, Div. of Applied Mathematics, Brown University, June 1970.

7. E. Hewitt, E. P. Wigner, On a theorem of magnus, Proc. Amer. Math. Soc., (1957), $740-744$.

8. L. H. Loomis, An Introduction to Abstract Harmonic Analysis, Van Nostrand, Princeton, New Jersey, 1953.

9. M. A. Naimark, Normed Rings, P. Noordhoff, Groningen, 1964.

10. W. Rudin, Fourier Analysis on Groups, Interscience, New York, 1962.

Received August 2, 1971 and in revised form October 28, 1971. This research was supported in part by NASA under Grant No. NGL 40-002-015 and the National Research Council of Canada. The results constitute a part of the authors doctoral dissertation written under the supervision of Professor P. L. Falb.

UNIVERSity OF BRITISH COLUMbia 


\section{PACIFIC JOURNAL OF MATHEMATICS}

\section{EDITORS}

\author{
H. SAMELSON \\ Stanford University \\ Stanford, California 94305 \\ C. R. HOBBY \\ University of Washington \\ Seattle, Washington 98105
}

\section{J. DugundjI}

Department of Mathematics University of Southern California Los Angeles, California 90007

\section{RICHARD ARENS}

University of California

Los Angeles, California 90024

\section{ASSOCIATE EDITORS}
E. F. BECKENBACH
B. H. NeumanN
F. WOLF
K. YosHIDA

\section{SUPPORTING INSTITUTIONS}

\author{
UNIVERSITY OF BRITISH COLUMBIA \\ CALIFORNIA INSTITUTE OF TECHNOLOGY \\ UNIVERSITY OF CALIFORNIA \\ MONTANA STATE UNIVERSITY \\ UNIVERSITY OF NEVADA \\ NEW MEXICO STATE UNIVERSITY \\ OREGON STATE UNIVERSITY \\ UNIVERSITY OF OREGON \\ OSAKA UNIVERSITY
}

\author{
UNIVERSITY OF SOUTHERN CALIFORNIA \\ STANFORD UNIVERSITY \\ UNIVERSITY OF TOKYO \\ UNIVERSITY OF UTAH \\ WASHINGTON STATE UNIVERSITY \\ UNIVERSITY OF WASHINGTON \\ ${ }^{*} \stackrel{*}{*}{ }^{*}$
AMERICAN MATHEMATICAL SOCIETY
NAVAL WEAPONS CENTER
}

The Supporting Institutions listed above contribute to the cost of publication of this Journal, but they are not owners or publishers and have no responsibility for its content or policies.

Mathematical papers intended for publication in the Pacific Journal of Mathematics should be in typed form or offset-reproduced, (not dittoed), double spaced with large margins. Underline Greek letters in red, German in green, and script in blue. The first paragraph or two must be capable of being used separately as a synopsis of the entire paper. The editorial "we" must not be used in the synopsis, and items of the bibliography should not be cited there unless absolutely necessary, in which case they must be identified by author and Journal, rather than by item number. Manuscripts, in duplicate if possible, may be sent to any one of the four editors. Please classify according to the scheme of Math. Rev. Index to Vol. 39. All other communications to the editors should be addressed to the managing editor, Richard Arens, University of California, Los Angeles, California, 90024.

50 reprints are provided free for each article; additional copies may be obtained at cost in multiples of 50 .

The Pacific Journal of Mathematics is published monthly. Effective with Volume 16 the price per volume (3 numbers) is $\$ 8.00$; single issues, $\$ 3.00$. Special price for current issues to individual faculty members of supporting institutions and to individual members of the American Mathematical Society: $\$ 4.00$ per volume; single issues $\$ 1.50$. Back numbers are available.

Subscriptions, orders for back numbers, and changes of address should be sent to Pacific Journal of Mathematics, 103 Highland Boulevard, Berke'ey, California, 94708.

PUBLISHED BY PACIFIC JOURNAL OF MATHEMATICS, A NON-PROFIT CORPORATION

Printed at Kokusai Bunken Insatsusha (International Academic Printing Co., Ltd.), 270, 3-chome Totsuka-cho, Shinjuku-ku, Tokyo 160, Japan. 


\section{Pacific Journal of Mathematics}

\section{Vol. 43, No. $3 \quad$ May, 1972}

Max K. Agoston, An obstruction to finding a fixed point free map on a manifold.... 543

Nadim A. Assad and William A. Kirk, Fixed point theorems for set-valued mappings

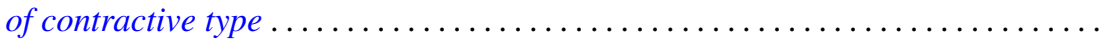

John Winston Bunce, Characterizations of amenable and strongly amenable

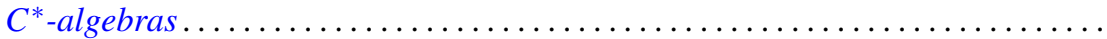

Erik Maurice Ellentuck and Alfred Berry Manaster, The decidability of a class of

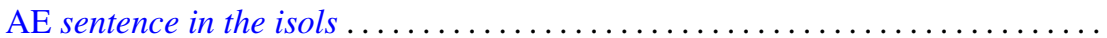

U. Haussmann, The inversion theorem and Plancherel's theorem in a Banach

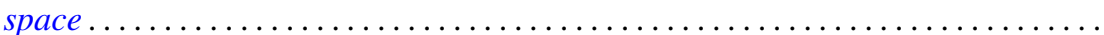

Peter Lawrence Falb and U. Haussmann, Bochner's theorem in infinite dimensions.

Peter Fletcher and William Lindgren, Quasi-uniformities with a transitive base ..... Dennis Garbanati and Robert Charles Thompson, Classes of unimodular abelian

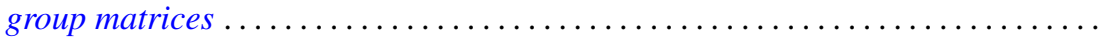

Kenneth Hardy and R. Grant Woods, On c-realcompact spaces and locally bounded normal functions

Manfred Knebusch, Alex I. Rosenberg and Roger P. Ware, Grothendieck and Witt rings of hermitian forms over Dedekind rings .......................

George M. Lewis, Cut loci of points at infinity.

Jerome Irving Malitz and William Nelson Reinhardt, A complete countable $L_{\omega_{1}}^{Q}$ theory with maximal models of many cardinalities . . . . . . . . . . . . . . . . .

Wilfred Dennis Pepe and William P. Ziemer, Slices, multiplicity, and Lebesgue

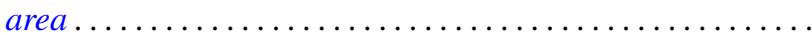

Keith Pierce, Amalgamating abelian ordered groups . .

Stephen James Pride, Residual properties of free groups . . . . . . . . . . . . . 725

Roy Martin Rakestraw, The convex cone of n-monotone functions .

T. Schwartzbauer, Entropy and approximation of measure preserving transformations .

Peter F. Stebe, Invariant functions of an iterative process for maximization of a polynomial...

Kondagunta Sundaresan and Wojbor Woyczynski, L-orthogonally scattered

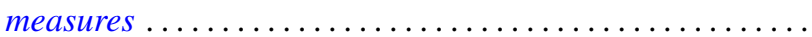

Kyle David Wallace, $C_{\lambda}$-groups and $\lambda$-basic subgroups $\ldots \ldots \ldots$

Barnet Mordecai Weinstock, Approximation by holomorphic functions on certain

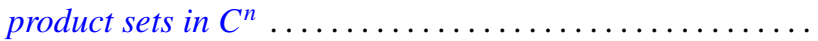

Donald Steven Passman, Corrections to: "Isomorphic groups and group rings”.

Don David Porter, Correction to: "Symplectic bordism, Stiefel-Whitney numbers, and a Novikov resolution"

John Ben Butler, Jr., Correction to: “Almost smooth perturbations of self-adjoint operators".

Constantine G. Lascarides, Correction to: "A study of certain sequence spaces of Maddox and a generalization of a theorem of Iyer" ...... ...

George A. Elliott, Correction to: "An extension of some results of takesaki in the reduction theory of von neumann algebras" ......................... 